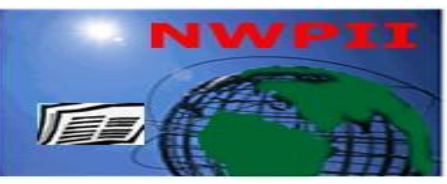

American Journal of Biomedical Sciences

ISSN: 1937-9080

nwpii.com/ajbms

\title{
Brain-Computer Interface Based on VEP and FMRI Package
}

\author{
Hadeel K. AlJobouri*, Faten Emad Ali
}

Biomedical Engineering Department, College of Engineering, Al-Nahrain University, Baghdad, IRAQ

"Corresponding Author

Dr. Hadeel K. AlJobouri

Biomedical Engineering Department

College of Engineering

Al-Nahrain University

Baghdad, IRAQ

Email: hadeel_bme77@yahoo.com

Received:08 December 2018 ; $\mid$ Revised:27 December 2018;| Accepted:15 March 2019

\section{Abstract}

A Brain-Computer Interface (BCI) is a construction that measures signals produced by the central nervous system (CNS) to perform the desired action or used for rehabilitation next stroke or any other disorders. In this paper, a new educational tool is designed and implemented using two functional data in the same package. Visual Evoked Potential (VEP) and functional Magnetic Resonance Imaging (fMRI) are designed in the same MATLAB-based Graphical User Interface (GUI). This work presented a new comparison between fMRI and VEP techniques.

Keywords: Brain-computer Interface (BCI); MATLAB-based Graphical User Interface (GUI); Visual Evoked Potential (VEP); Functional Magnetic Resonance Imaging (fMRI)

\section{Introduction}

A brain is the center of the human nervous system. Most of the activities of the body are controlled by the brain. All the information is coordinated, integrated and processed by the brain to convert the decisions as the commands to be sent to the rest of the body.

For the last decade, many obscurities are becoming clear with the expansion of cognitive neuroscience and the development of functional brain imaging techniques. These aim to recognize how the brain works in standings of its physiology, functional architecture and dynamics. Visual
Evoked Potential (VEP) and functional Magnetic Resonance Imaging (fMRI), are of the functional brain imaging techniques used in this work.

VEP is a non-invasive analysis method, gives objective information about the function of the entire vision structure. Using VEP can help distinguish between healthy and diseased eyes. VEP used to measure the complete visual pathway from the lens to the visual cortex, to identify mechanical or neural abnormalities connected to vision. VEP used to measure a patient's eye-brain feedback time. VEP is the electric signal registered in the occipital region in response to a visual stimulus ${ }^{[1]}$. 
The development of VEP started in 1934 when Adrian and Matthew recorded potential changes in the nerve ganglia under light stimulation ${ }^{[2]}$. In 1961, Ciganek developed the first nomenclature for EEG measurements after his previous results of studies of the human EEG response to flash stimulus ${ }^{[3]}$. In 1965, Rainer used a checkerboard stimulation to mark the difference in the electrical responses to describe human VEPs ${ }^{[4]}$. Halliday et al. ${ }^{[5,6]}$, presented the first clinical examinations using VEP. In 1972, they recorded the delayed VEPs in a retrobulbar neuritis patient. Until today, there are extensive and different research presented to develop VEP processes.

Atilla et al. ${ }^{[7]}$, estimate pattern electroretinography (PERG) and pattern visual evoked potential (VEP) for the differential optic nerve diseases in 2006. The results showed that the VEP amplitude is decreased significantly in ischaemic optic neuropathies, while the potential delay was more significant in patients with optic neuritis. Based on Brain-Computer Interface (BCI), there are different applications in biomedical field ${ }^{[8]}$. Friganović et al. presented the improvement of a steady-state visual evoked potential (SSVEP) in 2016. They designed a small arrangement using only one bipolar channel and three LED chessboards for the acquisition of raw EEG dataset [9].

FMRI is a diagnostic method used MRI to measure the variations in the blood level in the active area of the brain. It is used to examine the functional brain activity to detect the effects of stroke or other disorder which cannot be found with other imaging techniques ${ }^{[10]}$. Physicians and healthcare staff executes fMRI to determine which part of the brain is functionally activated such as thought, speech, movement or sensation, which is called brain mapping. Also, it can be used for following the growth and function of brain tumors.
In this work, the performance of VEP and fMRI are compared and introduced in a table; also the presented two functional brain imaging techniques have designed in a simple Graphical User Interface (GUI) package.

GUI has been implemented for different medical applications ${ }^{[11]}$. David et al. ${ }^{[12]}$, used a 3T fMRI for study subjects during visual activity. Aljobouri et al. ${ }^{[13]}$ introduced Biosignal Processing and Medical Imaging (BSPMI) software package which involves two main modules. Different biomedical signal processing is presented in the first component, such as ECG, EEG and EMG signals. Also, there are different biomedical images presented in the second component, such as X-ray, CT, MRI, and fMRI. BSPMI software package based on Graphical User Interface (GUI) application with MATLAB.

The paper is ordered as follows: in Section 2, systems designs and simulations with VEP and fMRI have been described; in Section 3, the details of the methodology and results have been presented; conclusions are in Section 4.

\section{Systems Designs And Simulations}

The approach system in this work is used as a prototype using two of the neuroscience data VEP and fMRI in the same package. The following sections describe each neuroscience technique.

\subsection{VEP Device}

The VEP device mainly consists of electrodes, stimulus and recording parameters, as shown in Fig.1. The stimulus parameters divided into flash and pattern stimulus which the last consists of pattern reversal and onset/offset stimulus. Recording parameters consist of analysis time and amplification and averaging systems.

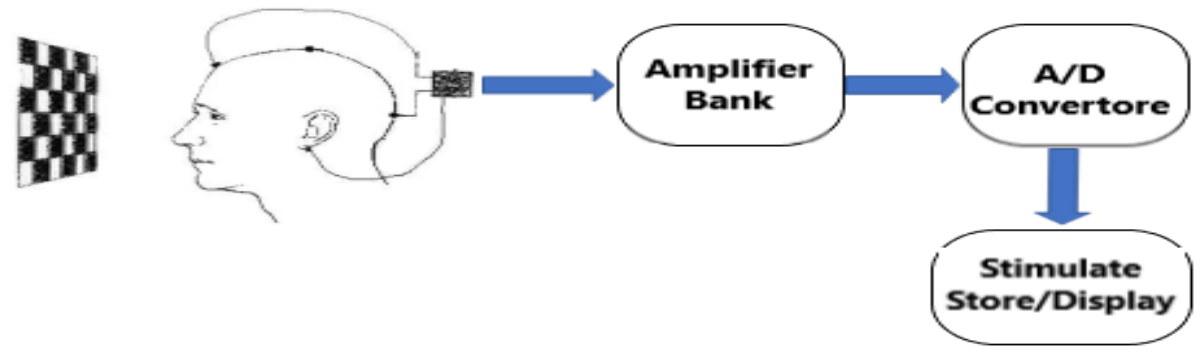

Figure 1: VEP Device parameters 
VEP using three electrodes that are the same as EEG electrodes [14]. Electrode placement correspondence to the international 10/20

arrangement for electrode placement is shown in Fig.2.

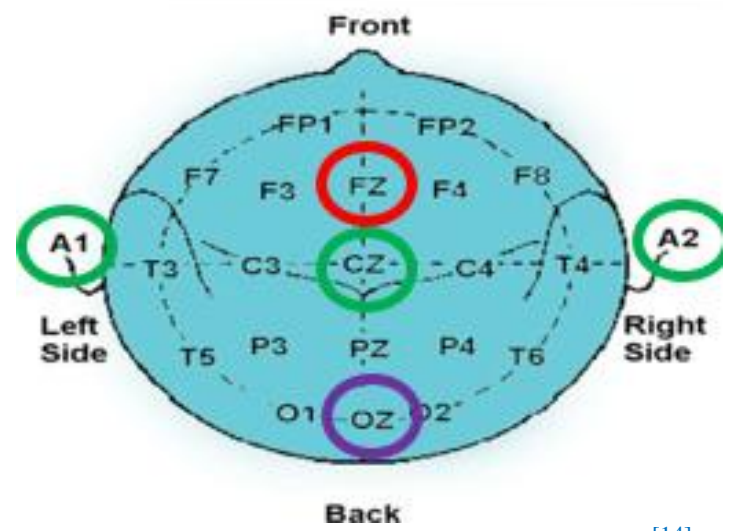

Figure 2: VEP Electrodes Placement ${ }^{[14]}$

Flash stimulus:

In flash stimulation the response to diffusely flashing light stimuli that subtends a visual field of 20 degrees as in Fig.3. Nowadays, the flash stimulus is infrequently used due to the high variability within and across subjects. On the other hand, it is the simple response than pattern VEP, merely indicates that light has been perceived by cortex shows little interocular asymmetry. This stimulus is helpful to use when testing babies or subjects with poor collaboration ${ }^{[15]}$.
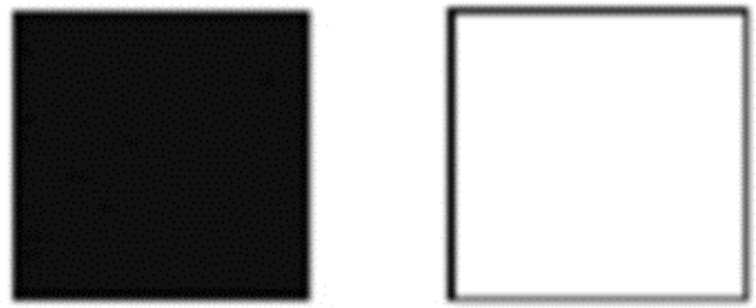

Figure 3: Flash stimulus ${ }^{[15]}$

Figure 4 illustrates the flash stimulation waveform in which the Time (latency) is measured in milliseconds (ms) and the Amplitude is measured in microvolts, the signal is characterized by a sequence of deflections labeled as negative and positive in a numerical order $(\mathrm{N} 1, \mathrm{P} 1, \mathrm{~N} 2, \mathrm{P} 2, \mathrm{~N} 3$, P3).

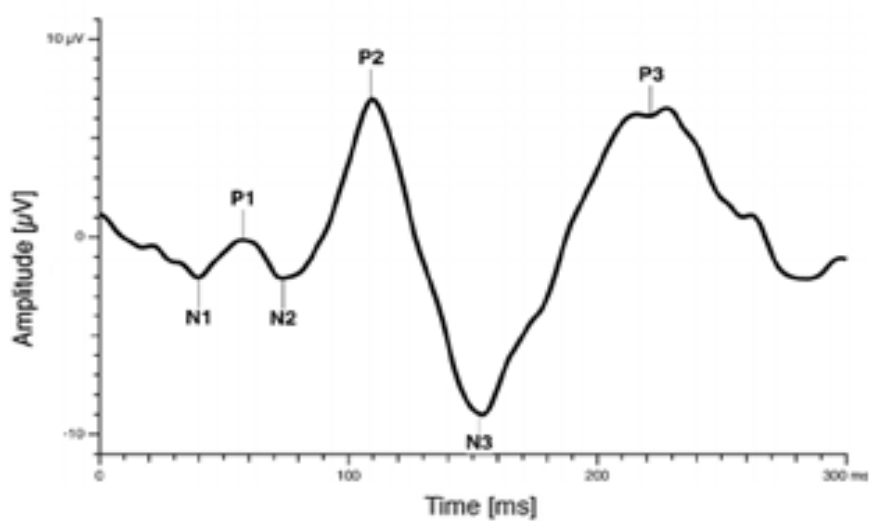

Figure 4: flash stimulation waveform 
Patterned stimulus:

Patterned stimulus is recommended, which is consists of a black and white checkerboard ${ }^{[16]}$. The stimulus is defined by the visual subtended angle by the side of a single check as shown in Fig.5.

Patterned stimulus consists of pattern reversal and onset/offset stimulus ${ }^{[16]}$.

Pattern reversal stimulus contains black and white checks, it changes phase (i.e., black to white and white to black) sharply and repetitively at a definite number of reversals per second as shown in Fig.6. The overall change in the luminance of the screen is not detectable. The stimulus should be defined in terms of the reversal frequency, the visual angle of each check, mean luminance the pattern contrast, number of reversals and field size [17].
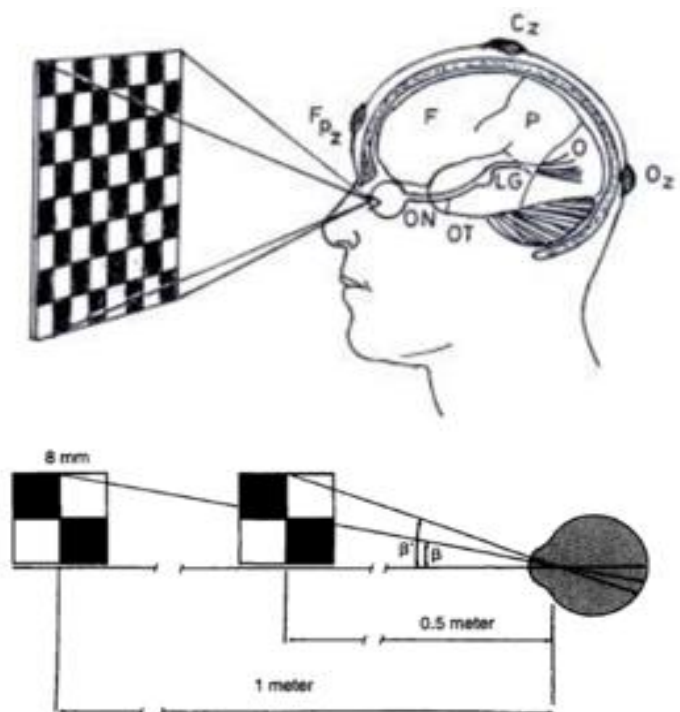

Figure 5: patterned stimulus ${ }^{[16]}$
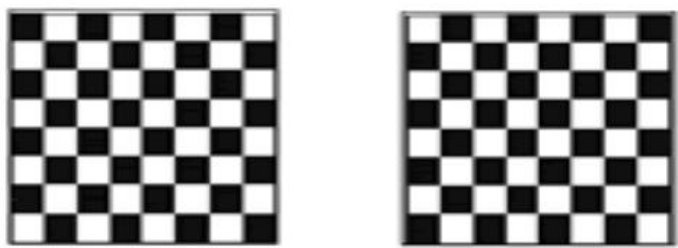

Figure 6: Pattern reversal stimulus ${ }^{[16]}$

Figure 7 illustrates the Pattern reversal stimulus waveform in which the Time (latency) is measured in milliseconds (ms) and the Amplitude is measured in microvolts and N75-100-N135
Complex where N75 indicate the negative pulse around $75 \mathrm{~ms}$ next the pattern onset, P100 indicate the positive pulse about $100 \mathrm{~ms}$ and N135 indicate the negative pulse around $135 \mathrm{~ms}$.

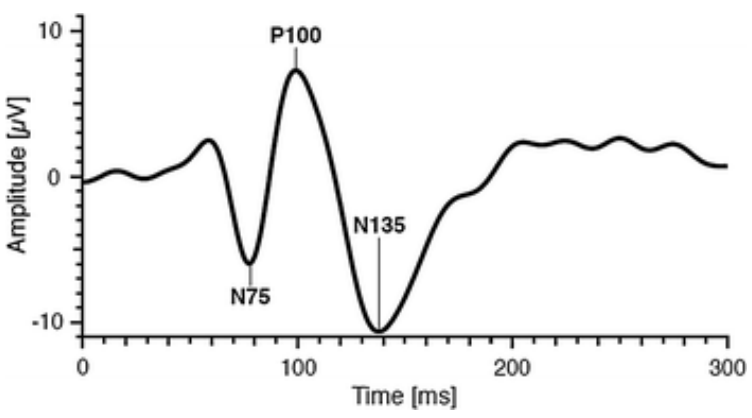

Figure 7: Pattern reversal stimulus wave 
In pattern onset/offset stimulus, the checkerboard pattern is sharply exchanged with a diffuse gray background as shown in Fig.8. The

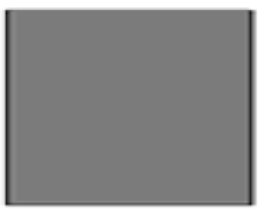

mean luminance of the diffuse background and the checkerboard must be matching ${ }^{[17]}$.

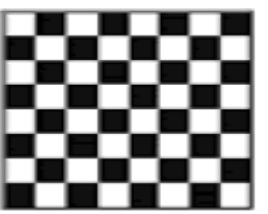

Figure 8: Pattern onset/offset stimulus ${ }^{[17]}$

Fig.9 illustrates the Pattern onset/offset stimulus waveform in which the Time (latency) is measured in milliseconds (ms) and the Amplitude is measured in microvolts and $\mathrm{C} 1-\mathrm{C} 2-\mathrm{C} 3$ Complex

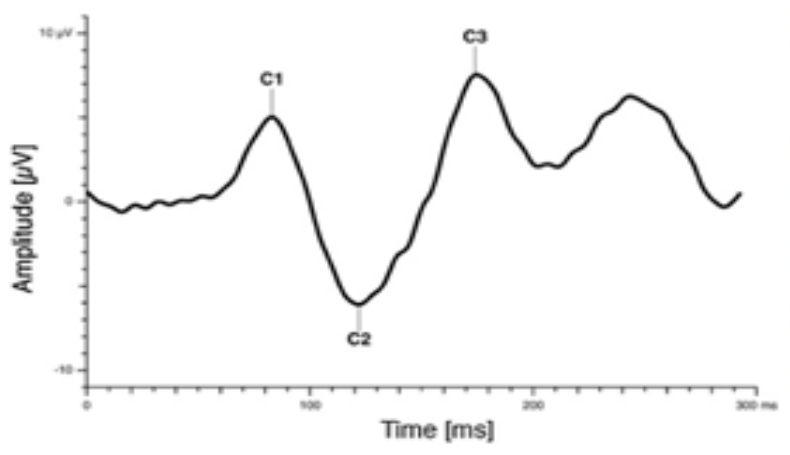

Figure 9: Pattern onset/offset stimulus wave

The grouping of amplitude and latency is useful in defining the health of the visual pathway. The Amplitude $(\mu \mathrm{v})$ indicates the extent of electrical energy connecting the visual cortex and quantity of "how much" information is connecting the visual cortex. While Latency (Peak Time) (ms) shows the time of the electrical signal passes from the retina to the visual cortex. The measurements can detect subjects affecting vision if it proceeds more time for the electrical signals to influence the visual cortex. where $\mathrm{C} 1$ indicates the positive around $75 \mathrm{~ms}, \mathrm{C} 2$ indicates the negative around $125 \mathrm{~ms}$ and $\mathrm{C} 3$ indicate the positive around $150 \mathrm{~ms}$.

\subsection{FMRI System}

Functional magnetic resonance imaging (fMRI) is a sturdy technique to check brain activity by identifying fluctuations with blood flow. Blood oxygenation level dependent (BOLD) signal demonstrate the changes in the level of $\mathrm{O} 2$ in the blood, which is directly concerned with the activity of the neuron. Modeling the Hemodynamic Response Function (HRF) is a decisive step in fMRI studies of brain activity, and it is often desirable to estimate HRF parameters physiologically ${ }^{[18]}$.

Fig.10 shows the HRF of experimental auditory paradigm for sequential alternated between rest and auditory stimulation.

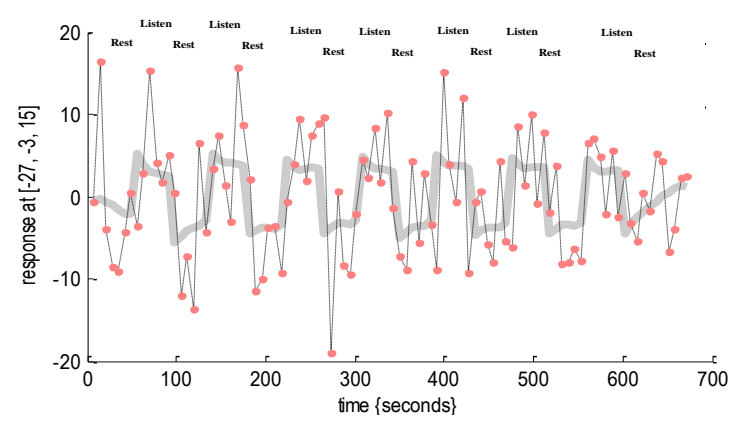

Figure 10: Experimental paradigm "silence" and "talk" "[19] 
FMRI is utilized to comprehend neuronal mechanisms beyond numerous defects, for example, Alzheimer's disease, autism spectrum disorders, Parkinson's disease, schizophrenia, and bipolar disorder. The fMRI data is obtained from a scanner machine as raw data in arrangements of 3D images on account of the varieties of voxel intensities over time. By various experimental conditions, the obtained fMRI data are shaped like a set of BOLD signal variations and noises or artifacts. The noises are ascribed to the system hardware such as the MRI scanner, the subject movement (e.g., head movement), or physiological effects ${ }^{[19]}$.

\section{Methodology and results}

In this study, a new brain-computer interfacing model based on VEP and fMRI is designed and implemented as a software package based on GUI using MATLAB.

A new comparison between VEP and fMRI is introduced in this work. The differences between VEP and fMRI in the field of vision tested can be listed in a table (1).

Table 1: Comparison of VEP with fMRI

VEP

fMRI

\begin{tabular}{|c|c|}
\hline $\begin{array}{c}\text { measures visual pathway activity by } \\
\text { electrical activity of the visual cortex }\end{array}$ & $\begin{array}{c}\text { measures visual pathway activity by measuring } \\
\text { fluctuations with blood flow }\end{array}$ \\
\hline Higher temporal resolution & Lower temporal resolution \\
\hline Lower spatial resolution & Higher spatial resolution \\
\hline Measure optical nerve activity directly & Measure optical nerve activity indirectly (BOLD) \\
\hline Accessible cost for most researchers. & Cost device \\
\hline Can be fixed or portable. & Not portable \\
\hline The output results as waves. & The output result as image \\
\hline
\end{tabular}

Fig.11 shows the presented functional software package. The toolbox gives a feature of displaying VEP and fMRI in the same window. There is a measurement analysis panel; presented in the suggested model. The data graph can be saved as a .FIG, .PNG, .TIF, .BMP; and it can be printed directly. When the graph is saved, different modifications can be done for these graphs using image analysis techniques.

The used VEP dataset in this toolbox from male subject ${ }^{[20]}$. The VEP sample rate is 1.200192 $\mathrm{kHz}$ (833.2 usec/point) for 6002 points, the vertical scale is $795.3585 \mathrm{nV} \mathrm{V/div}(\max 7.953585 \mathrm{uV})$ and the horizontal scale is $10 \mathrm{~Hz} / \mathrm{div}$ (146.5078 $\mathrm{mHz}$ /point) starting at $0 \mathrm{~Hz}$.

The used fMRI dataset in this toolbox from a repetition priming experiment implemented using event-related fMRI. The acquired images were using continuous Echo-Planar Imaging (EPI). The EPI was with 24 descendant slices (64X64 3X3 $\mathrm{mm} 2$ ). The thickness and gap of each slice were 3 and $1.5 \mathrm{~mm}$ respectively. The echo time (TE) and repetition time (TR) were $40 \mathrm{~ms}$ and $2 \mathrm{~s}$ respectively. The presented study is a $2 \mathrm{X} 2$ factorial, with "fame" and "repetition" factors. Famous and non-famous faces were viewed twice against a checkerboard baseline ${ }^{[21]}$. The subject was requested to create fame decisions by making key presses. The first and second appearances of famous and non-famous faces made four events-types of interest.

In this work, the healthcare staff and biomedical research can use these two functional data at the same time and compare the results easily. The users can load data easily using only a few buttons as shown in Fig.11, so it not needs any software programmer just deals with buttons. 


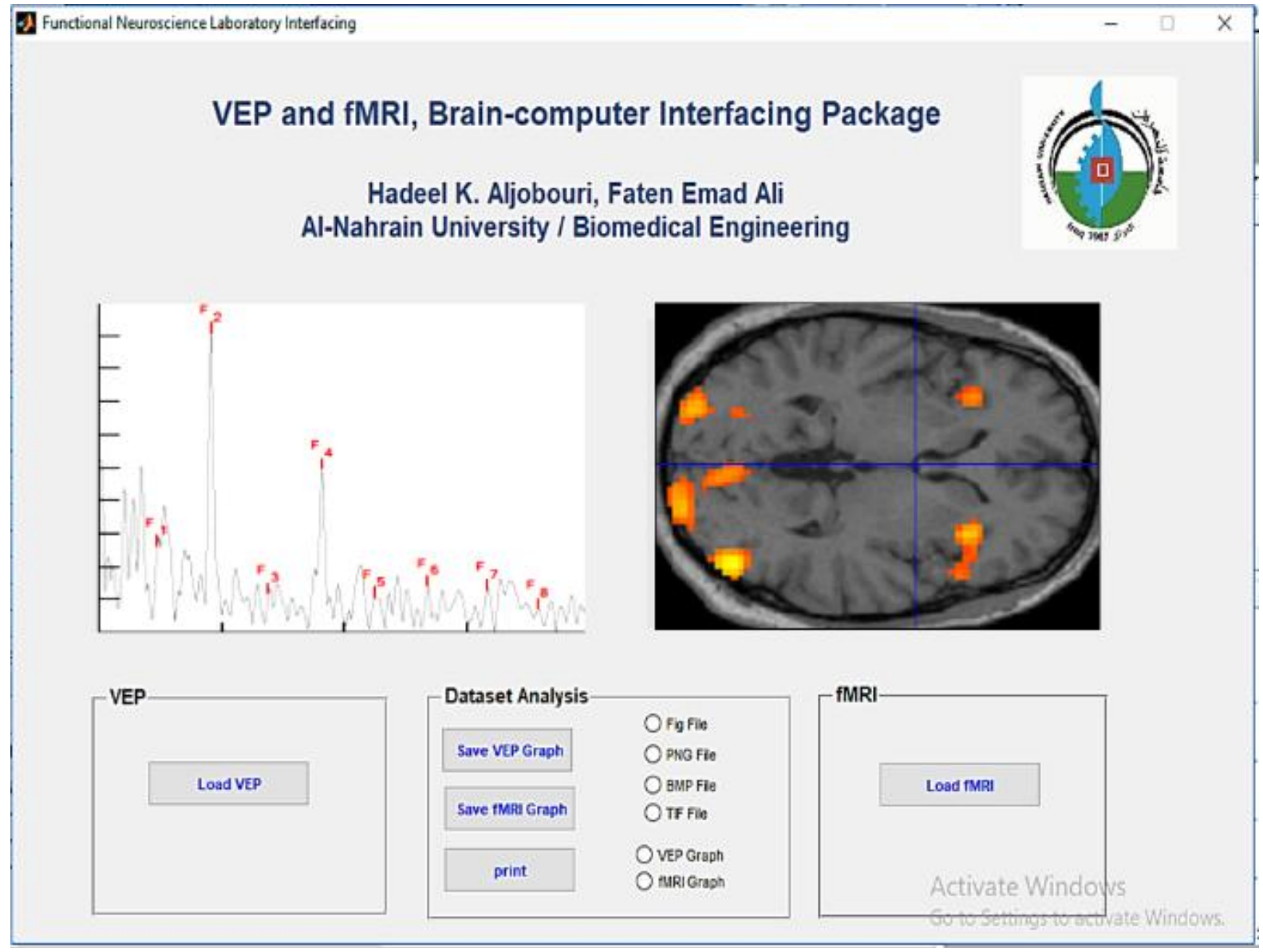

Figure 11: Functional Imaging Software Package

\section{Conclusion}

A new brain-computer interface has introduced in this work. BCI is a direct statement pathway between the human brain and a peripheral device. Two of the functional vision responsible techniques fMRI and VEP are designed and implemented in the same simple software package based on GUI using MATLAB. Also, a new comparison between fMRI and VEP techniques is summarized in this work. There is no toolbox presented previously between fMRI and VEP. So, the presented work will be the first in the literature.

The designed package submitted a userfriendly graphical interface as part of biomedical engineering experimental laboratories. The functional GUI can be simply used by students and researchers in the engineering laboratories. Without installing MATLAB program, the user can deal with this software using only the standalone application or executable file (exe file).
For future, different medical images analysis techniques can be introduced as a new panel in the same software package.

\section{References}

1 von dem Hagen EA, Hoffmann MB, Morland AB. Identifying human albinism: a comparison of VEP and fMRI. Invest Ophthalmol Vis Sci 2008; 49(1): 238-249 [PMID: 18172098 DOI: 10.1167/iovs.07-0458]

2 Adrian E.D., Matthews B.H.C. The Interpretation of Potential Waves in the Cortex. Physiological Laboratory, Cambridge, the Journal of Physiology, 1934.

3 Ciganek L. The EEG response (evoked potential) to light stimulus in man. Electroencephalogr Clin Neurophysiol 1961; 13: 165-172 [PMID: 13693643] 
4 Spehlmann R. The averaged electrical responses to diffuse and to patterned light in the human. Electroencephalogr Clin Neurophysiol 1965; 19(6): 560-569 [PMID: 4158632]

5 Halliday AM, McDonald WI, Mushin J. Delayed visual evoked response in optic neuritis. Lancet 1972; 1(7758): 982-985 [PMID: 4112367]

6 Halliday AM, McDonald WI, Mushin J. Visual evoked response in diagnosis of multiple sclerosis. Br Med J 1973; 4(5893): 661-664 [PMID: 4758547 PMCID: PMC1587677]

7 Atilla H, Tekeli O, Ornek K, Batioglu F, Elhan AH, Eryilmaz T. Pattern electroretinography and visual evoked potentials in optic nerve diseases. J Clin Neurosci 2006; 13(1): 55-59 [PMID: $16410198 \quad$ DOI: 10.1016/j.jocn.2005.02.007]

8 Aly A. F., Agameia A., Eldesouky A. S., M. Sharaf A. Scaffold Development and Characterization Using CAD System. American Journal of Biomedical Sciences. 2011; 3(4): 268-277.

9 Friganović K., Medved M., Cifrek M. Braincomputer Interface Based on Steady-state Visual Evoked Potentials. University of Zagreb, Faculty of Electrical Engineering and Computing, Croatia, 2016.

10 Radiological Society of North America. Magnetic Resonance, Functional (fMRI) Brain. RadiologyInfo.org, pp.1-8, 2018

11 Ahmed T., Dutta J. C. A GUI (Graphical User Interface) framework to Introduce H-H Model in Comparison with Kv3.3 Model. American Journal of Biomedical Sciences. 2014; 6(1): 1-5.

12 Somers DC, Dale AM, Seiffert AE, Tootell RB. Functional MRI reveals spatially specific attentional modulation in human primary visual cortex. Proc Natl Acad Sci U S A 1999; 96(4): 1663-1668 [PMID: 9990081 PMCID: PMC15552]
13 AlJobouri H. K., Alziarjawey H. A., I. Çankaya. Biosignal Processing, Medical Imaging and fMRI (BSPMI) Software Package Based on MATLAB GUI for Education and Research. International Journal of Scientific Research in Information Systems and Engineering. 2015, 1(2): 2380-8128.

14 Odom J., Bach M. Visual evoked potentials standard. Documental Ophthalmologica, Kluwer Academic Publishers, Netherlands, 2004; 108: 115-123.

15 Laura M. Flash visual evoked potentials and early visual development in infants born to drug misusing mothers. McGlone, MD thesis; 2012.

16 Sarojini. B.K, Basavaraj S., and Mukartihal G.B. Autoregressive Modeling of Visual Evoked Potentials and Its Applications to Optic Nerve Diseases-Ischemic Optic Neuropathy and Optic Neuritis. International Journal on Recent and Innovation Trends in Computing and Communication. 2016, 4(2): 110-114.

17 Pitzalis S, Strappini F, De Gasperis M, Bultrini A, Di Russo F. Spatio-temporal brain mapping of motion-onset VEPs combined with fMRI and retinotopic maps. PLoS One 2012; 7(4): e35771 DOI: 10.1371/journal.pone.0035771

18 Aljobouri H.K., Cankaya I., Karal O. From biomedical signal processing techniques to fMRI parcellation. Bioscience. Biotechnology

Research Asia. 2015; 12: 1115-1138.

19 Aljobouri HK, Jaber HA, Kocak OM, Algin O, Cankaya I. Clustering fMRI data with a robust unsupervised learning algorithm for neuroscience data mining. $\boldsymbol{J}$ Neurosci Methods 2018; 299: 45-54 [PMID: 29471065 DOI: 10.1016/j.jneumeth.2018.02.007]

20 Retrieved May 31, 2016, from https://catalog.data.gov/dataset?tags=visualevoked-potentials, by Will Boyes on EP3

21 Retrieved May 13, 1991, from https://www.fil.ion.ucl.ac.uk/spm/data/face_re p/. 1991 